\title{
Pion-photon and photon-pion transition form factors in light-cone formalism
}

\author{
Bo-Wen Xiao \\ Department of Physics, Peking University, Beijing 100871, China \\ Bo-Qiang Ma* \\ CCAST (World Laboratory), P.O. Box 8730, Beijing 100080, China \\ Department of Physics, Peking University, Beijing 100871, China
}

\begin{abstract}
We derive the minimal Fock-state expansions of the pion and the photon wave functions in lightcone formalism, then we calculate the pion-photon and the photon-pion transition form factors of $\gamma^{*} \pi^{0} \rightarrow \gamma$ and $\gamma^{*} \gamma \rightarrow \pi^{0}$ processes by employing these quark-antiquark wave functions of the pion and the photon. We find that our calculation for the $\gamma^{*} \gamma \rightarrow \pi^{0}$ transition form factor agrees with the experimental data at low and moderately high energy scale. Moreover, the physical differences and inherent connections between the transition form factors of $\gamma^{*} \pi^{0} \rightarrow \gamma$ and $\gamma^{*} \gamma \rightarrow \pi^{0}$ have been illustrated, which indicate that these two physical processes are intrinsically related. In addition, we also discuss the $\pi^{0} \rightarrow \gamma \gamma$ form factor and the decay width $\Gamma(\pi \rightarrow \gamma \gamma)$ at $Q^{2}=0$.
\end{abstract}

PACS numbers: 14.40.Aq; 12.39.Ki; 13.40.Gp; 13.60.Le

*Electronic address: mabq@phy.pku.edu.cn corresponding author. 


\section{INTRODUCTION}

The light-cone formalism provides a convenient framework for the relativistic description of hadrons in terms of quark and gluon degrees of freedom [1]. In this formalism, the hadronic wave function which describes a hadronic composite state at a particular light-cone time $\tau$ is expressed in terms of a series of light-cone wave functions in Fock-state basis, for example, for the pion

$$
|\pi\rangle=\sum|q \bar{q}\rangle \psi_{q \bar{q}}+\sum|q \bar{q} g\rangle \psi_{q \bar{q} g}+\cdots
$$

and the temporal evolution of the state is generated by the light-cone Hamiltonian $H_{L C}^{Q C D}$. Similar with $\pi^{0}$, we assume that the photon may also have this kind of Fock-state expansion

$$
|\gamma\rangle=\sum|q \bar{q}\rangle \psi_{q \bar{q}}
$$

for application to QCD involved processes. To simplify the problem, we only take into account the minimal Fock-states of the pion and the photon, which are the lowest valence states of their light-cone wave functions and the first order contributions in the calculation. This consideration will be proved reasonable by the good agreement between the numerical results and the experimental data.

We will derive the quark-antiquark wave functions of the pion and the photon by employing the light-cone Fock expansion of the minimal Fock-states. Brodsky et al. [2, 3] have discussed the relativistic QED composite systems by giving explicit the light-cone wave functions for the two-particle Fock-states of the electron in QED. Along with the similar idea, we try to obtain the Fock expansions of the photon and the pion wave functions by calculating their vertexes in this paper. After that, we discuss the pion-photon $\left(\gamma^{*} \pi^{0} \rightarrow \gamma\right)$ and the photon-pion $\left(\gamma^{*} \gamma \rightarrow \pi^{0}\right)$ transition form factors in the light-cone quark model. There are many other different approaches to discuss these two transition form factors, such as the perturbative QCD formalism by Brodsky-Lepage [4] and Cao-Huang-Ma [5], as well as a light-front quark model by Kroll-Raulfs [6] for the $\gamma^{*} \pi^{0} \rightarrow \gamma$ transition, the QCD sum

rule calculation by Radyushkin-Ruskov [7], and a light-front quark model by Hwang 8] for the $\gamma^{*} \gamma \rightarrow \pi^{0}$ transition form factor. However, few people notice that these two transition form factors are physically different although they have inherent connections. In this work, we also try to elucidate the intrinsic relations and differences between these two cases in the light-cone formalism, thus arrive at a more clear understanding on the transition form 
factors of $\gamma^{*} \pi^{0} \rightarrow \gamma$ and $\gamma^{*} \gamma \rightarrow \pi^{0}$ processes.

The paper is organized as follows. In Sec. II and Sec. III, we will derive the pion and the photon wave functions with helicity structure by the relativistic vertex method of calculating the light-cone matrix elements that developed in Refs. [2, 3]. In Sec. IV, we obtain the pionphoton and the photon-pion transition form factors by using the pion-quark-antiquark and the photon-quark-antiquark wave functions. In Sec. V, we present a brief summary.

\section{THE TWO PARTICLE FOCK-STATE OF THE PION VERTEX}

We now derive the minimal Fock-state wave function of the pion in light-cone formalism. From the Fock-state basis of the pion vertex Eq. (11), we will employ two different methods to get the quark-antiquark wave function of the pion in this part of the paper. One is from the light-cone quark model by taking into account the Melosh-Wigner rotation effect [9, 10, 11], and another is from a full relativistic field theory treatment of the interaction vertex [2, 3]. It will be shown that the results from these two methods are essentially identical.

In the light-cone quark model, the light-cone wave function of a composite system can be obtained by transforming the ordinary equal-time (instant-form) wave function in the rest frame into that in the light-front dynamics, by taking into account the relativistic effects such as the Melosh-Wigner rotation [9, 10, 11, 12, 13, 14]. In the pion rest frame $\left(q_{1}+q_{2}=0\right)$, the ordinary $\mathrm{SU}(6)$ quark model spin space wave function of the pion is

$$
\chi_{T}=\left(\chi_{1}^{\uparrow} \chi_{2}^{\downarrow}-\chi_{2}^{\uparrow} \chi_{1}^{\downarrow}\right) / \sqrt{2}
$$

in which $\chi_{i}^{\uparrow \downarrow}$ is the two-component Pauli spinor and the two quarks have 4-momentum $q_{1}^{\mu}=\left(q_{1}^{0}, \mathbf{q}\right)$ and $q_{2}^{\mu}=\left(q_{2}^{0},-\mathbf{q}\right)$, with $q_{i}^{0}=\left(m_{i}^{2}+\mathbf{q}^{2}\right)^{1 / 2}$, respectively. The instant-form spin states $|J, s\rangle_{T}$ and the light-front spin states $|J, \lambda\rangle_{F}$ are related by the Melosh-Wigner rotation $U^{J}$ [15, 16$]$

$$
|J, \lambda\rangle_{F}=\sum_{s} U_{s \lambda}^{J}|J, s\rangle_{T}
$$

Applying the transformation Eq. (4) on both sides of Eq. (3), we can obtain the spin space wave function of the pion in the infinite-momentum frame. For the left side, i.e., the pion, the transformation is simple since the Melosh-Wigner rotation is unity. For the right side, i.e., the two spin-1/2 partons, the instance-form and the light-front spin states are related 
by the Melosh-Wigner transformation [15, 16, 17],

$$
\left\{\begin{array}{l}
\chi_{i}^{\uparrow}(T)=\omega_{i}\left[\left(q_{i}^{+}+m_{i}\right) \chi_{i}^{\uparrow}(F)-q_{i}^{R} \chi_{i}^{\downarrow}(F)\right], \\
\chi_{i}^{\downarrow}(T)=\omega_{i}\left[\left(q_{i}^{+}+m_{i}\right) \chi_{i}^{\downarrow}(F)+q_{i}^{L} \chi_{i}^{\uparrow}(F)\right],
\end{array}\right.
$$

where $\omega_{i}=\left[2 q_{i}^{+}\left(q_{i}^{0}+m_{i}\right)\right]^{-1 / 2}, q_{i}^{R, L}=q_{i}^{1} \pm \mathrm{i} q_{i}^{2}$, and $q_{i}^{+}=q_{i}^{0}+q_{i}^{3}$. Then we get the light-cone spin wave function of the pion $\left(m_{1}=m_{2}=m\right)$,

$$
\chi^{K}\left(x, \mathbf{k}_{\perp}\right)=\sum_{\lambda_{1}, \lambda_{2}} C_{0}^{F}\left(x, \mathbf{k}_{\perp}, \lambda_{1}, \lambda_{2}\right) \chi_{1}^{\lambda_{1}}(F) \chi_{2}^{\lambda_{2}}(F)
$$

where the component coefficients $C_{J=0}^{F}\left(x, \mathbf{k}_{\perp}, \lambda_{1}, \lambda_{2}\right)$, when expressed in terms of the instantform momentum $q_{\mu}=\left(q^{0}, \mathbf{q}=\mathbf{k}\right)$, have the forms:

$$
\left\{\begin{array}{l}
C_{0}^{F}\left(x, \mathbf{k}_{\perp}, \uparrow, \downarrow\right)=+\omega_{1} \omega_{2}\left[\left(q_{1}^{+}+m_{1}\right)\left(q_{2}^{+}+m_{2}\right)-\mathbf{q}_{\perp}^{2}\right] / \sqrt{2}, \\
C_{0}^{F}\left(x, \mathbf{k}_{\perp}, \downarrow, \uparrow\right)=-\omega_{1} \omega_{2}\left[\left(q_{1}^{+}+m_{1}\right)\left(q_{2}^{+}+m_{2}\right)-\mathbf{q}_{\perp}^{2}\right] / \sqrt{2}, \\
C_{0}^{F}\left(x, \mathbf{k}_{\perp}, \uparrow, \uparrow\right)=\omega_{1} \omega_{2}\left[\left(q_{1}^{+}+m_{1}\right) q_{2}^{L}-\left(q_{2}^{+}+m_{2}\right) q_{1}^{L}\right] / \sqrt{2} \\
C_{0}^{F}\left(x, \mathbf{k}_{\perp}, \downarrow, \downarrow\right)=\omega_{1} \omega_{2}\left[\left(q_{1}^{+}+m_{1}\right) q_{2}^{R}-\left(q_{2}^{+}+m_{2}\right) q_{1}^{R}\right] / \sqrt{2}
\end{array}\right.
$$

which satisfy the relation,

$$
\sum_{\lambda_{1}, \lambda_{2}} C_{0}^{F}\left(x, \mathbf{k}_{\perp}, \lambda_{1}, \lambda_{2}\right)^{*} C_{0}^{F}\left(x, \mathbf{k}_{\perp}, \lambda_{1}, \lambda_{2}\right)=1
$$

We can see that there are also two higher helicity $\left(\lambda_{1}+\lambda_{2}= \pm 1\right)$ components in the expression of the light-cone spin wave function of the pion besides the ordinary helicity $\left(\lambda_{1}+\lambda_{2}=0\right)$ components. Such higher helicity components [9, 10, 18, 19] come from the Melosh-Wigner rotation, and the same effect plays an important role to understand the proton "spin puzzle" in the nucleon case [17, 20]. One may also state that these higher helicity components contain contribution from orbital angular moment from a relativistic viewpoint 21]. Therefore we can obtain the light-cone representation for the spin structure of the pion, which is the minimal Fock-state of the pion light-cone wave function:

$$
\left\{\begin{array}{l}
\Psi_{\pi R}\left(x, \mathbf{k}_{\perp}, \uparrow, \downarrow\right)=+\frac{m}{\sqrt{2\left(m^{2}+\mathbf{k}_{\perp}^{2}\right)}} \varphi_{\pi},\left[l^{z}=0\right] \\
\Psi_{\pi R}\left(x, \mathbf{k}_{\perp}, \downarrow, \uparrow\right)=-\frac{m}{\sqrt{2\left(m^{2}+\mathbf{k}_{\perp}^{2}\right)}} \varphi_{\pi},\left[l^{z}=0\right] \\
\Psi_{\pi R}\left(x, \mathbf{k}_{\perp}, \uparrow, \uparrow\right)=-\frac{k_{1}-i k_{2}}{\sqrt{2\left(m^{2}+\mathbf{k}_{\perp}^{2}\right)}} \varphi_{\pi},\left[l^{z}=-1\right] \\
\Psi_{\pi R}\left(x, \mathbf{k}_{\perp}, \downarrow, \downarrow\right)=-\frac{k_{1}+i k_{2}}{\sqrt{2\left(m^{2}+\mathbf{k}_{\perp}^{2}\right)}} \varphi_{\pi},\left[l^{z}=+1\right]
\end{array}\right.
$$


in which we may employ the Brodsky-Huang-Lepage (BHL) prescription [22],

$$
\varphi_{\pi}(x, \mathbf{k})=A \exp \left[-\frac{1}{8 \beta^{2}} \frac{\mathbf{k}_{\perp}^{2}+m^{2}}{x(1-x)}\right]
$$

for the momentum space wave function. Each configuration satisfies the spin sum rule: $J^{z}=S_{q}^{z}+S_{\bar{q}}^{z}+l^{z}=0$. Hence, the Fock expansion of the two particle Fock-state for the pion has four possible spin combinations:

$$
\begin{aligned}
\left|\Psi_{\pi}\left(P^{+}, \mathbf{P}_{\perp}=\mathbf{0}_{\perp}\right)\right\rangle= & \int \frac{\mathrm{d}^{2} \mathbf{k}_{\perp} \mathrm{d} x}{16 \pi^{3}} \\
& \times\left[\Psi_{\pi R}\left(x, \mathbf{k}_{\perp}, \uparrow, \downarrow\right)\left|x P^{+}, \mathbf{k}_{\perp}, \uparrow, \downarrow\right\rangle+\Psi_{\pi R}\left(x, \mathbf{k}_{\perp}, \downarrow, \uparrow\right)\left|x P^{+}, \mathbf{k}_{\perp}, \downarrow, \uparrow\right\rangle\right. \\
& \left.+\Psi_{\pi R}\left(x, \mathbf{k}_{\perp}, \uparrow, \uparrow\right)\left|x P^{+}, \mathbf{k}_{\perp}, \uparrow, \uparrow\right\rangle+\Psi_{\pi R}\left(x, \mathbf{k}_{\perp}, \downarrow, \downarrow\right)\left|x P^{+}, \mathbf{k}_{\perp}, \downarrow, \downarrow\right\rangle\right] .
\end{aligned}
$$

To get the Fock-state spin wave function of the pion, we adopt here another simple way in the full relativistic field theory treatment of the interaction vertex along with the idea in 22, 3]. We assume that one can consider the pion vertex connecting to two spin- $\frac{1}{2}$ fermions (e.g. two quarks) by only taking into account the minimal Fock-state. In the standard light-cone frame for the pion form factor,

$$
\left\{\begin{aligned}
p_{1} & =\left(x P^{+}, \frac{\mathbf{p}_{1 \perp}^{2}+m^{2}}{x P^{+}}, \mathbf{p}_{1 \perp}\right) \\
p_{2} & =\left((1-x) P^{+}, \frac{\mathbf{p}_{2 \perp}^{2}+m^{2}}{(1-x) P^{+}}, \mathbf{p}_{2 \perp}\right) \\
P & =\left(P^{+}, \frac{M^{2}}{P^{+}}, \mathbf{0}_{\perp}\right) \\
P^{\prime} & =\left(P^{\prime+}, \frac{q^{2}+\mathbf{q}_{\perp}^{2}}{P^{\prime+}}, \mathbf{p}_{\perp}^{\prime}\right) \\
q & =\left(0, \frac{Q^{2}}{P^{+}}, \mathbf{q}_{\perp}\right)
\end{aligned}\right.
$$

we can obtain the above four components of the spin wave function by calculating the matrix elements of

$$
\frac{\bar{u}\left(p_{1}^{+}, p_{1}^{-}, \mathbf{k}_{\perp}\right)}{\sqrt{p_{1}^{+}}} \gamma_{5} \frac{v\left(p_{2}^{+}, p_{2}^{-},-\mathbf{k}_{\perp}\right)}{\sqrt{p_{2}^{+}}},
$$

from which we have

$$
\left\{\begin{array}{l}
\frac{\bar{u}_{\uparrow}}{\sqrt{p_{1}^{+}}} \gamma_{5} \frac{v_{\downarrow}}{\sqrt{p_{2}^{+}}}=+\frac{2 m P^{+}}{4 m x(1-x) P^{+2}}, \\
\frac{\bar{u}_{\downarrow}}{\sqrt{p_{1}^{+}}} \gamma_{5} \frac{v_{\uparrow}}{\sqrt{p_{2}^{+}}}=-\frac{2 m P^{+}}{4 m x(1-x) P^{+2}}, \\
\frac{\bar{u}_{\uparrow}}{\sqrt{p_{1}^{+}}} \gamma_{5} \frac{v_{\uparrow}}{\sqrt{p_{2}^{\prime+}}}=-\frac{2\left(k_{1}-i k_{2}\right) P^{+}}{4 m x(1-x) P^{+2}}, \\
\frac{\bar{u}_{\downarrow}}{\sqrt{p_{1}^{+}}} \gamma_{5} \frac{v_{\downarrow}}{\sqrt{p_{2}^{+}}}=-\frac{2\left(k_{1}+i k_{2}\right) P^{+}}{4 m x(1-x) P^{+2}} .
\end{array}\right.
$$

After the normalization, we can get the same result of Eq. (9) too. 
Furthermore, we can get $\left\langle\Psi_{\pi}\left(P^{+}, \mathbf{P}_{\perp}=\mathbf{0}_{\perp}\right)\right|$ from the matrix elements of

$$
\frac{\bar{v}\left(p_{2}^{+}, p_{2}^{-},-\mathbf{k}_{\perp}\right)}{\sqrt{p_{2}^{+}}} \gamma_{5} \frac{u\left(p_{1}^{+}, p_{1}^{-}, \mathbf{k}_{\perp}\right)}{\sqrt{p_{1}^{+}}}
$$

so that

$$
\begin{aligned}
\left\langle\Psi_{\pi}\left(P^{+}, \mathbf{P}_{\perp}\right)\right|= & \int \frac{\mathrm{d}^{2} \mathbf{k}_{\perp} \mathrm{d} x}{16 \pi^{3}} \\
& \times\left[\Psi_{\pi L}\left(x, \mathbf{k}_{\perp}, \uparrow, \downarrow\right)\left\langle x P^{+}, \mathbf{k}_{\perp}, \uparrow, \downarrow\right|+\Psi_{\pi L}\left(x, \mathbf{k}_{\perp}, \downarrow, \uparrow\right)\left\langle x P^{+}, \mathbf{k}_{\perp}, \downarrow, \uparrow\right|\right. \\
& \left.+\Psi_{\pi L}\left(x, \mathbf{k}_{\perp}, \uparrow, \uparrow\right)\left\langle x P^{+}, \mathbf{k}_{\perp}, \uparrow, \uparrow\right|+\Psi_{\pi L}\left(x, \mathbf{k}_{\perp}, \downarrow, \downarrow\right)\left\langle x P^{+}, \mathbf{k}_{\perp}, \downarrow, \downarrow\right|\right]
\end{aligned}
$$

in which,

$$
\left\{\begin{array}{l}
\Psi_{\pi L}\left(x, \mathbf{k}_{\perp}, \uparrow, \downarrow\right)=-\frac{m}{\sqrt{2\left(m^{2}+\mathbf{k}_{\perp}^{2}\right)}} \varphi_{\pi}^{*}, \\
\Psi_{\pi L}\left(x, \mathbf{k}_{\perp}, \downarrow, \uparrow\right)=+\frac{m}{\sqrt{2\left(m^{2}+\mathbf{k}_{\perp}^{2}\right)}} \varphi_{\pi}^{*}, \\
\Psi_{\pi L}\left(x, \mathbf{k}_{\perp}, \uparrow, \uparrow\right)=+\frac{k_{1}+i k_{2}}{\sqrt{2\left(m^{2}+\mathbf{k}_{\perp}^{2}\right)}} \varphi_{\pi}^{*}, \\
\Psi_{\pi L}\left(x, \mathbf{k}_{\perp}, \downarrow, \downarrow\right)=+\frac{k_{1}-i k_{2}}{\sqrt{2\left(m^{2}+\mathbf{k}_{\perp}^{2}\right)}} \varphi_{\pi}^{*} .
\end{array}\right.
$$

Therefore, we can get the pion elastic charge form factor through the definition: $\left\langle\Psi_{\pi}\left(P^{\prime}\right)\left|J^{+}\right| \Psi_{\pi}(P)\right\rangle \delta^{3}\left(\mathbf{p}+\mathbf{q}-\mathbf{p}^{\prime}\right)=F_{\pi}\left(Q^{2}\right)\left(P+P^{\prime}\right)$, where $J^{\mu}=\bar{q} e \gamma^{\mu} e_{q} q$ is the vector current,

$$
F_{\pi^{+}}\left(Q^{2}\right)=\left(e_{u}+e_{\bar{d}}\right) \int_{0}^{1} \mathrm{~d} x \int \frac{\mathrm{d}^{2} \mathbf{k}_{\perp}}{16 \pi^{3}} \frac{m^{2}+\mathbf{k}_{\perp} \cdot \mathbf{k}_{\perp}^{\prime}}{\sqrt{m^{2}+\mathbf{k}_{\perp}^{2}} \sqrt{m^{2}+\mathbf{k}_{\perp}^{\prime 2}}} \varphi_{\pi}^{*}\left(x, \mathbf{k}_{\perp}^{\prime}\right) \varphi_{\pi}\left(x, \mathbf{k}_{\perp}\right),
$$

in which $\mathbf{k}_{\perp}^{\prime}=\mathbf{k}_{\perp}+(1-x) \mathbf{q}_{\perp}$ for the final state light-cone wave function when taking into account the Drell-Yan-West assignment [23].

\section{THE TWO PARTICLE FOCK-STATE OF THE PHOTON VERTEX}

Similar with the pion vertex and with the same assumption, we can also obtain the spin wave function of the spin-1 photon from the minimal Fock-state basis of the photon vertex Eq. (2) by calculating the matrix elements of

$$
\frac{\bar{v}\left(p_{2}^{+}, p_{2}^{-}, \mathbf{p}_{2 \perp}\right)}{\sqrt{p_{2}^{+}}} \gamma \cdot \epsilon^{*} \frac{u\left(p_{1}^{+}, p_{1}^{-}, \mathbf{p}_{1 \perp}\right)}{\sqrt{p_{1}^{+}}},
$$

which are the numerators of the wave functions corresponding to each constituent spin $S^{z}$ configuration. The two boson polarization vectors in light-cone gauge are $\epsilon^{\mu}=\left(\epsilon^{+}=\right.$ 
$\left.0, \epsilon^{-}, \epsilon_{\perp}\right)$, where $\epsilon_{\perp \uparrow, \downarrow}=\mp \frac{1}{\sqrt{2}}(\widehat{\mathbf{x}} \pm \widehat{\mathbf{y}})$. To satisfy the Lorentz condition $k_{\text {photon }} \cdot \epsilon=0$, the polarizations have the relation $\epsilon^{-}=\frac{2 \epsilon_{\perp} \cdot \mathbf{k}_{\perp}}{k^{+}}$with $k_{\text {hoton }}$,

$$
\begin{cases}\Psi_{L}^{\uparrow}\left(x, \mathbf{k}_{\perp}, \uparrow, \downarrow\right)=-\frac{\sqrt{2}\left(k_{1}-i k_{2}\right)}{1-x} \varphi_{\gamma}, & {\left[l^{z}=+1\right]} \\ \Psi_{L}^{\uparrow}\left(x, \mathbf{k}_{\perp}, \downarrow, \uparrow\right)=+\frac{\sqrt{2}\left(k_{1}-i k_{2}\right)}{x} \varphi_{\gamma}, & {\left[l^{z}=+1\right]} \\ \Psi_{L}^{\uparrow}\left(x, \mathbf{k}_{\perp}, \uparrow, \uparrow\right)=-\frac{\sqrt{2} m}{x(1-x)} \varphi_{\gamma}, & {\left[l^{z}=0\right]} \\ \Psi_{L}^{\uparrow}\left(x, \mathbf{k}_{\perp}, \downarrow, \downarrow\right)=0, & \end{cases}
$$

in which:

$$
\varphi_{\gamma}=\frac{e_{q}}{D}=\frac{e_{q}}{\lambda^{2}-\frac{m^{2}+\mathbf{k}_{\perp}^{2}}{x}-\frac{m^{2}+\mathbf{k}_{\perp}^{2}}{1-x}},
$$

where $\lambda$ is the photon mass and equals to 0 . Moreover, as Lepage and Brodsky had mentioned in [4], the transition form factor of the process $\gamma^{*} \gamma \rightarrow \pi^{0}$ has the energy denominator

$$
D=\lambda^{2}-\frac{m^{2}+\mathbf{k}_{\perp}^{\prime 2}}{x}-\frac{m^{2}+\mathbf{k}_{\perp}^{\prime 2}}{1-x}
$$

where $\mathbf{k}_{\perp}^{\prime}=\mathbf{k}_{\perp}+(1-x) \mathbf{q}_{\perp}$ for the final state light-cone wave function when taking into account the Drell-Yan-West assignment [23]. Each configuration satisfies the spin sum rule: $J^{z}=S_{q}^{z}+S_{\bar{q}}^{z}+l^{z}=+1$. Therefore, the two particle Fock-state for the photon $\left(J^{z}=+1\right)$ has four possible spin combinations:

$$
\begin{aligned}
\left\langle\Psi_{\gamma}^{\uparrow}\left(P^{\prime+}, \mathbf{P}_{\perp}^{\prime}\right)\right|= & \int \frac{\mathrm{d}^{2} \mathbf{k}_{\perp} \mathrm{d} x}{16 \pi^{3}} \\
& \times\left[\Psi_{L}^{\uparrow}\left(x, \mathbf{k}_{\perp}, \uparrow, \downarrow\right)\left\langle x P^{\prime+}, \mathbf{k}_{\perp}, \uparrow, \downarrow\right|+\Psi_{L}^{\uparrow}\left(x, \mathbf{k}_{\perp}, \downarrow, \uparrow\right)\left\langle x P^{\prime+}, \mathbf{k}_{\perp}, \downarrow, \uparrow\right|\right. \\
& \left.+\Psi_{L}^{\uparrow}\left(x, \mathbf{k}_{\perp}, \uparrow, \uparrow\right)\left\langle x P^{\prime+}, \mathbf{k}_{\perp}, \uparrow, \uparrow\right|+\Psi_{L}^{\uparrow}\left(x, \mathbf{k}_{\perp}, \downarrow, \downarrow\right)\left\langle x P^{\prime+}, \mathbf{k}_{\perp}, \downarrow, \downarrow\right|\right],
\end{aligned}
$$

and respectively we can get the wave function of the photon which $J^{z}=-1$,

$$
\left\{\begin{array}{l}
\Psi_{L}^{\downarrow}\left(x, \mathbf{k}_{\perp}, \uparrow, \downarrow\right)=-\frac{\sqrt{2}\left(k_{1}+i k_{2}\right)}{x} \varphi_{\gamma},\left[l^{z}=-1\right] \\
\Psi_{L}^{\downarrow}\left(x, \mathbf{k}_{\perp}, \downarrow, \uparrow\right)=+\frac{\sqrt{2}\left(k_{1}+i k_{2}\right)}{1-x} \varphi_{\gamma},\left[l^{z}=-1\right] \\
\Psi_{L}^{\downarrow}\left(x, \mathbf{k}_{\perp}, \uparrow, \uparrow\right)=0, \\
\Psi_{L}^{\downarrow}\left(x, \mathbf{k}_{\perp}, \downarrow, \downarrow\right)=-\frac{\sqrt{2} m}{x(1-x)} \varphi_{\gamma}, \quad\left[l^{z}=0\right]
\end{array}\right.
$$




$$
\begin{aligned}
\left\langle\Psi_{\gamma}^{\downarrow}\left(P^{\prime+}, \mathbf{P}_{\perp}^{\prime}\right)\right|= & \int \frac{\mathrm{d}^{2} \mathbf{k}_{\perp} \mathrm{d} x}{16 \pi^{3}} \\
& \times\left[\Psi_{L}^{\downarrow}\left(x, \mathbf{k}_{\perp}, \uparrow, \downarrow\right)\left\langle x P^{\prime+}, \mathbf{k}_{\perp}, \uparrow, \downarrow\right|+\Psi_{L}^{\downarrow}\left(x, \mathbf{k}_{\perp}, \downarrow, \uparrow\right)\left\langle x P^{\prime+}, \mathbf{k}_{\perp}, \downarrow, \uparrow\right|\right. \\
& \left.+\Psi_{L}^{\downarrow}\left(x, \mathbf{k}_{\perp}, \uparrow, \uparrow\right)\left\langle x P^{\prime+}, \mathbf{k}_{\perp}, \uparrow, \uparrow\right|+\Psi_{L}^{\downarrow}\left(x, \mathbf{k}_{\perp}, \downarrow, \downarrow\right)\left\langle x P^{\prime+}, \mathbf{k}_{\perp}, \downarrow, \downarrow\right|\right] .
\end{aligned}
$$

By calculating the matrix elements of

$$
\frac{\bar{u}\left(p_{1}^{+}, p_{1}^{-}, \mathbf{p}_{1 \perp}\right)}{\sqrt{p_{1}^{+}}} \gamma \cdot \epsilon \frac{v\left(p_{2}^{+}, p_{2}^{-}, \mathbf{p}_{2 \perp}\right)}{\sqrt{p_{2}^{+}}}
$$

we obtain $\left|\Psi_{\gamma}^{\uparrow}\left(P^{\prime+}, \mathbf{P}_{\perp}^{\prime}\right)\right\rangle$, which is the conjugate part of $\left\langle\Psi_{\gamma}^{\uparrow}\left(P^{\prime+}, \mathbf{P}_{\perp}^{\prime}\right)\right|$

$$
\begin{gathered}
\\
\left\{\begin{array}{l}
\Psi_{R}^{\uparrow}\left(x, \mathbf{k}_{\perp}, \uparrow, \downarrow\right)=-\frac{\sqrt{2}\left(k_{1}+i k_{2}\right)}{1-x} \varphi_{\gamma}, \\
\Psi_{R}^{\uparrow}\left(x, \mathbf{k}_{\perp}, \downarrow, \uparrow\right)=+\frac{\sqrt{2}\left(k_{1}+i k_{2}\right)}{x} \varphi_{\gamma}, \\
\Psi_{R}^{\uparrow}\left(x, \mathbf{k}_{\perp}, \uparrow, \uparrow\right)=-\frac{\sqrt{2} m}{x(1-x)} \varphi_{\gamma}, \\
\Psi_{R}^{\uparrow}\left(x, \mathbf{k}_{\perp}, \downarrow, \downarrow\right)=0,
\end{array}\right. \\
\left|\Psi_{\gamma}^{\uparrow}\left(P^{\prime+}, \mathbf{P}_{\perp}^{\prime}\right)\right\rangle=\int \frac{\mathrm{d}^{2} \mathbf{k}_{\perp} \mathrm{d} x}{16 \pi^{3}} \\
\times\left[\Psi_{R}^{\uparrow}\left(x, \mathbf{k}_{\perp}, \uparrow, \downarrow\right)\left|x P^{\prime+}, \mathbf{k}_{\perp}, \uparrow, \downarrow\right\rangle+\Psi_{R}^{\uparrow}\left(x, \mathbf{k}_{\perp}, \downarrow, \uparrow\right)\left|x P^{\prime+}, \mathbf{k}_{\perp}, \downarrow, \uparrow\right\rangle\right. \\
\left.+\Psi_{R}^{\uparrow}\left(x, \mathbf{k}_{\perp}, \uparrow, \uparrow\right)\left|x P^{\prime+}, \mathbf{k}_{\perp}, \uparrow, \uparrow\right\rangle+\Psi_{R}^{\uparrow}\left(x, \mathbf{k}_{\perp}, \downarrow, \downarrow\right)\left|x P^{\prime+}, \mathbf{k}_{\perp}, \downarrow, \downarrow\right\rangle\right] .
\end{gathered}
$$

From above calculations, we can arrive at the following conclusions: when a composite system is transformed from an ordinary equal-time frame to a light-cone frame, the spin of each constituent will undergo a Melosh-Wigner rotation, and these spin rotations for the constituents are not necessarily the same since the constituents have different internal motions. Therefore, the sum of the constituents' spin is not Lorentz invariant. For example, although the pion has only the $\lambda_{1}+\lambda_{2}=0$ spin components in the rest frame of itself, it may have $\lambda_{1}+\lambda_{2}= \pm 1$ spin components in the light-cone frame (infinite-momentum frame), in which $\lambda_{1}$ and $\lambda_{2}$ are the quark and the antiquark helicities, respectively. We could also obtain the similar results in the Fock expansion of the photon wave function, in which there are the $\lambda_{1}+\lambda_{2}=0$ helicity states for a vector particle in the light-cone frame. The similar 
conclusion is also true in pure QED case of the electron as a composite system of two Fockstate particles 3]. These general results for the spin structure of composite systems are distinct from the naive intuitive expectation that the quark spins sum to the hadron spin, and support the viewpoint that the proton "spin puzzle" can be understood as due to the relativistic motion of quarks inside the nucleon in the light-cone formalism [17, 20].

\section{PION-PHOTON AND PHOTON-PION TRANSITION FORM FACTORS}

In this part, we study the pion-photon and the photon-pion transition form factors, and perform our theoretical and numerical analysis respectively. In addition, we discuss the $\pi^{0} \rightarrow \gamma \gamma$ form factor and the decay width $\Gamma(\pi \rightarrow \gamma \gamma)$ at $Q^{2}=0$. As a matter of fact, we can find that $F_{\gamma^{*} \pi \rightarrow \gamma}(0)=F_{\gamma^{*} \gamma \rightarrow \pi}(0)=F_{\pi \rightarrow \gamma \gamma}(0)$ for these three processes at $Q^{2}=0$. Theoretically and generally speaking, the transition form factor calculated by BrodskyLepage [4], Cao-Huang-Ma [5], and Kroll-Raulfs [6] should be the pion-photon transition form factor $\gamma^{*} \pi^{0} \rightarrow \gamma$ in the physical process $e+\pi^{0} \rightarrow e+\gamma$, as $e \rightarrow e$ provides the virtual photon. This should be physically different from the photon-pion transition form factor $\gamma^{*} \gamma \rightarrow \pi^{0}$ as can be realized in $e+e \rightarrow e+e+\pi^{0}$ or $e+A$ (Nucleus) $\rightarrow e+A+\pi^{0}$. The

$\gamma^{*} \gamma \rightarrow \pi^{0}$ transition form factor has been measured at Cornell [24] and at DESY [25] through the $e^{+}+e^{-} \rightarrow e^{+}+e^{-}+\pi^{0}$ process, while the latter process $e+A$ (Nucleus) $\rightarrow e+A+\pi^{0}$ can be performed by the HERMES Collaboration or other facilities. By employing these experimental data, Hwang and Choi-Ji discussed the transition form factors $\gamma^{*} \gamma \rightarrow \pi^{0}$ and $\gamma^{*} \gamma^{*} \rightarrow \pi^{0}$ theoretically in [8].

\section{A. $\gamma^{*} \pi^{0} \rightarrow \gamma$ TRANSITION FORM FACTOR}

From the pion and the photon vertexes in form of wave functions that we have got, we can naturally obtain the $\gamma^{*} \pi^{0} \rightarrow \gamma$ transition form factor from its definition. The form factor $F_{\gamma^{*} \pi \rightarrow \gamma}$, in which a pion is struck by an off-shell photon and decays into an on-shell photon, is defined by the $\gamma^{*} \pi \gamma$ vertex,

$$
\Gamma_{\mu}=-i e^{2} F_{\gamma^{*} \pi \rightarrow \gamma}\left(Q^{2}\right) \varepsilon_{\mu \nu \rho \sigma} p_{\pi}^{\nu} \epsilon^{\rho} q^{\sigma}
$$

in which $q$ is the momentum of the off-shell photon, $-Q^{2}=q^{2}=q^{+} q^{-}-\mathbf{q}_{\perp}^{2}=-\mathbf{q}_{\perp}^{2}$, and $\epsilon$ is the polarization vector of the on-shell photon. 
For the physical state of $\pi^{0}$, one should also take into account the color and flavor degrees of freedom into account [4, 5]

$$
\left|\Psi_{\pi^{0}}\right\rangle=\sum_{a} \frac{\delta_{b}^{a}}{\sqrt{n_{c}}} \frac{1}{\sqrt{2}}\left[\left|u^{a} \bar{u}^{b}\right\rangle-\left|d^{a} \bar{d}^{b}\right\rangle\right],
$$

where $a$ and $b$ are color indices, $n_{c}=3$ is the number of colors, and now $\left|q^{a} \bar{q}^{b}\right\rangle$ contains the full spin structure in Sec. II. Therefore, we can get

$$
\Gamma^{+}=\left\langle\Psi_{\gamma}^{\uparrow}\left(P^{\prime+}, \mathbf{P}_{\perp}^{\prime}\right)\left|J^{+}\right| \Psi_{\pi}\left(P^{+}, \mathbf{P}_{\perp}\right)\right\rangle \delta^{3}\left(\mathbf{p}+\mathbf{q}-\mathbf{p}^{\prime}\right)
$$

As a matter of fact, we can get the same result if we use $\left\langle\Psi_{\gamma}^{\downarrow}\left(P^{\prime+}, \mathbf{P}_{\perp}^{\prime}\right)\right|$. Then we get:

$$
\begin{aligned}
F_{\gamma^{*} \pi \rightarrow \gamma}\left(Q^{2}\right)= & \frac{\Gamma^{+}}{-i e^{2}\left(\epsilon_{\perp} \times \mathbf{q}_{\perp}\right) p_{\pi}^{-}} \\
= & 2 \sqrt{3}\left(e_{u}^{2}-e_{d}^{2}\right) \int_{0}^{1} \mathrm{~d} x \int \frac{\mathrm{d}^{2} \mathbf{k}_{\perp}}{16 \pi^{3}} \varphi_{\pi}\left(x, \mathbf{k}_{\perp}\right) \\
& \left\{\frac{m}{x \sqrt{m^{2}+\mathbf{k}_{\perp}^{2}}} \times\left[\frac{1}{\frac{m^{2}+\mathbf{k}_{\perp}^{\prime 2}}{x}+\frac{m^{2}+\mathbf{k}_{\perp}^{\prime 2}}{1-x}}\right]+(1 \leftrightarrow 2)\right\} \\
= & 4 \sqrt{3}\left(e_{u}^{2}-e_{d}^{2}\right) \int_{0}^{1} \mathrm{~d} x \int \frac{\mathrm{d}^{2} \mathbf{k}_{\perp}}{16 \pi^{3}} \varphi_{\pi}\left(x, \mathbf{k}_{\perp}\right) \\
& \frac{m}{x \sqrt{m^{2}+\mathbf{k}_{\perp}^{2}}} \times\left[\frac{1}{\frac{m^{2}+\mathbf{k}_{\perp}^{\prime 2}}{x}+\frac{m^{2}+\mathbf{k}_{\perp}^{\prime 2}}{1-x}}\right] .
\end{aligned}
$$

The arguments of the final states of the light-cone wavefunction are $\mathbf{k}_{\perp i}^{\prime}=\mathbf{k}_{\perp i}+\mathbf{q}_{\perp}-x_{i} \mathbf{q}_{\perp}$ for the struck quark, $\mathbf{k}_{\perp ;}^{\prime}=\mathbf{k}_{\perp i}-x_{i} \mathbf{q}_{\perp}$ for the spectator quark after considering the DrellYan-West assignment [23], and the virtual photon momentum $q_{\mu}$ is specified with $q^{+}=0$ to eliminate the Z-graph contributions. Therefore, we can obtain the internal quark transverse momentum of the struck pion $\mathbf{k}_{\perp}^{\prime}=\mathbf{k}_{\perp}+(1-x) \mathbf{q}_{\perp}$ in the center of mass frame. For the struck particle of the process, we should employ the Drell-Yan-West assignment in the derivation of the formula for the transition form factor. The reason is that if we do not know the wave function of a moving particle (the struck particle after the virtual photon vertex), we can adopt the wave function in the rest frame of this particle after using the Drell-Yan-West assignment. Hence, the rest frame wave functions for the struck particle both before and after the virtual photon vertex can be used in the calculation of the transition form factor, even if the struck particle is in a rest frame before the virtual photon vertex and in a moving frame after the virtual photon vertex. 
Furthermore, we can obtain the $\gamma^{*} \pi^{0} \rightarrow \gamma^{*}$ transition form factor by substituting a virtual photon $\gamma^{*}$ for the on-shell photon $\gamma$, which means to substitute $-Q^{\prime 2}$ for $\lambda^{2}$ and gives:

$$
\begin{aligned}
F_{\gamma^{*} \pi \rightarrow \gamma^{*}}\left(Q^{2}, Q^{\prime 2}\right)= & 4 \sqrt{3}\left(e_{u}^{2}-e_{d}^{2}\right) \int_{0}^{1} \mathrm{~d} x \int \frac{\mathrm{d}^{2} \mathbf{k}_{\perp}}{16 \pi^{3}} \varphi_{\pi}\left(x, \mathbf{k}_{\perp}\right) \frac{m}{x \sqrt{m^{2}+\mathbf{k}_{\perp}^{2}}} \\
& \times\left[\frac{1}{Q^{\prime 2}+\frac{m^{2}+\mathbf{k}_{\perp}^{\prime 2}}{x}+\frac{m^{2}+\mathbf{k}_{\perp}^{\prime 2}}{1-x}}\right] .
\end{aligned}
$$

The leading order behavior of $F_{\gamma^{*} \pi \rightarrow \gamma^{*}}\left(Q^{2}\right)$ can be obtained by taking limits of $Q^{2} \rightarrow \infty$ and $Q^{\prime 2} \rightarrow \infty$, thus we can get:

$$
F_{\gamma^{*} \pi \rightarrow \gamma^{*}}\left(Q^{2}, Q^{\prime 2}\right)=4 \sqrt{3}\left(e_{u}^{2}-e_{d}^{2}\right) \int_{0}^{1} \mathrm{~d} x \phi_{\pi}(x) \frac{1}{x Q^{\prime 2}+(1-x) Q^{2}},
$$

which is equivalent to the results given in [5].

\section{B. $\pi^{0} \rightarrow \gamma \gamma$ FORM FACTOR AND DECAY WIDTH $\Gamma(\pi \rightarrow \gamma \gamma)$}

From general consideration, the $\pi^{0} \rightarrow \gamma \gamma$ form factor can not be calculated in the $q^{+}=0$ frame. Other choice of $q_{\mu}$ will cause contribution from Z-graphs, and it should give the same result as that in the $q^{+}=0$ case if all graphs are taken into account [26]. Therefore some more complicated diagrams should be included. Fortunately, we find that $F_{\pi \rightarrow \gamma \gamma}(0)=F_{\gamma \gamma^{*} \rightarrow \pi}(0)$ when $Q^{2}=0$. It is well known that $F_{\pi \rightarrow \gamma \gamma}(0)$ is related to the two photon partial decay width of pion $\Gamma(\pi \rightarrow \gamma \gamma)$ by [24]:

$$
\left|F_{\pi \rightarrow \gamma \gamma}(0)\right|^{2}=\frac{64 \pi \Gamma(\pi \rightarrow \gamma \gamma)}{(4 \pi \alpha)^{2} M_{\pi}^{3}}
$$

where $\alpha$ is the QED coupling constant, $M_{\pi}$ is the pion mass and $\Gamma(\pi \rightarrow \gamma \gamma)$ is the twophoton partial width of the pion. In addition, we can obtain:

$$
\begin{aligned}
F_{\gamma \gamma^{*} \rightarrow \pi}(0)= & F_{\pi \rightarrow \gamma \gamma}(0)=4 \sqrt{3}\left(e_{u}^{2}-e_{d}^{2}\right) \int_{0}^{1} \mathrm{~d} x \int \frac{\mathrm{d}^{2} \mathbf{k}_{\perp}}{16 \pi^{3}} \\
& {\left[\varphi_{\pi}\left(x, \mathbf{k}_{\perp}\right) \frac{m}{x \sqrt{m^{2}+\mathbf{k}_{\perp}^{2}}} \times \frac{1}{\frac{m^{2}+\mathbf{k}_{\perp}^{2}}{x}+\frac{m^{2}+\mathbf{k}_{\perp}^{2}}{1-x}}\right] . }
\end{aligned}
$$

Hence, we can get $\Gamma(\pi \rightarrow \gamma \gamma)$ from above calculations. 


\section{C. $\gamma^{*} \gamma \rightarrow \pi^{0}$ TRANSITION FORM FACTOR}

By using the Fock expansions of the pion and the photon wave functions that we have got above, we can naturally obtain the $\gamma^{*} \gamma \rightarrow \pi^{0}$ transition form factor from its definition. The form factor $F_{\gamma^{*} \gamma \rightarrow \pi^{0}}$, in which an on-shell photon is struck by an off-shell photon and decays into a pion, is defined by the $\gamma^{*} \gamma \pi$ vertex,

$$
\Gamma_{\mu}=-i e^{2} F_{\gamma^{*} \gamma \rightarrow \pi^{0}}\left(Q^{2}\right) \varepsilon_{\mu \nu \rho \sigma} p_{\pi}^{\nu} \epsilon^{\rho} q^{\sigma},
$$

in which $q$ is the momentum of the off-shell photon, $-Q^{2}=q^{2}=q^{+} q^{-}-\mathbf{q}_{\perp}^{2}=-\mathbf{q}_{\perp}^{2}$, and $\epsilon$ is the polarization vector of the on-shell photon. In the light-cone frame:

$$
\left\{\begin{array}{l}
p_{1}=\left(x P^{+}, \frac{\mathbf{k}_{\perp}^{2}+m^{2}}{x P^{+}}, \mathbf{k}_{\perp}\right) \\
p_{2}=\left((1-x) P^{+}, \frac{\mathbf{k}_{\perp}^{2}+m^{2}}{(1-x) P^{+}},-\mathbf{k}_{\perp}\right) \\
p_{1}^{\prime}=\left(x P^{\prime+}, \frac{\mathbf{k}_{\perp}^{\prime 2}+m^{2}}{x P^{+}}, \mathbf{k}_{\perp}^{\prime}\right) \\
P_{\gamma}=\left(P^{+}, \frac{q^{2}+\mathbf{q}_{\perp}^{2}}{P^{+}}, \mathbf{0}_{\perp}\right) \\
P_{\pi}^{\prime}=\left(P^{\prime+}, \frac{M^{2}}{P^{\prime}}, \mathbf{q}_{\perp}\right) \\
q=\left(0, \frac{Q^{2}}{P^{+}}, \mathbf{q}_{\perp}\right)
\end{array}\right.
$$

so we can get

$$
\begin{aligned}
F_{\gamma \gamma^{*} \rightarrow \pi}\left(Q^{2}\right)= & \frac{\Gamma^{+}}{-i e^{2}\left(\epsilon_{\perp} \times \mathbf{q}_{\perp}\right) p_{\pi}^{\prime-}} \\
= & -4 \sqrt{3}\left(e_{u}^{2}-e_{d}^{2}\right) \int_{0}^{1} \mathrm{~d} x \int \frac{\mathrm{d}^{2} \mathbf{k}_{\perp}}{16 \pi^{3}}\left[\varphi_{\pi}\left(x, \mathbf{k}_{\perp}^{\prime}\right) \frac{m}{x \sqrt{m^{2}+\mathbf{k}_{\perp}^{\prime 2}}}\right. \\
& \left.\times \frac{1}{\lambda^{2}-\frac{m^{2}+\mathbf{k}_{\perp}^{2}}{x}-\frac{m^{2}+\mathbf{k}_{\perp}^{2}}{1-x}}\right],
\end{aligned}
$$

in which $\mathbf{k}_{\perp}^{\prime}=\mathbf{k}_{\perp}+(1-x) \mathbf{q}_{\perp}$ after considering the Drell-Yan-West formula, and $\lambda(=0)$ is the photon mass.

Moreover, we can get the equivalent $F_{\gamma \gamma^{*} \pi}\left(Q^{2}\right)$ if we choose another different light-cone frame:

$$
\left\{\begin{array}{l}
p_{1}=\left(x P^{+}, \frac{\left(x \mathbf{P}_{\perp}+\mathbf{k}_{\perp}\right)^{2}+m^{2}}{x P^{+}}, x \mathbf{P}_{\perp}+\mathbf{k}_{\perp}\right) \\
p_{2}=\left((1-x) P^{+}, \frac{\left((1-x) \mathbf{P}_{\perp}-\mathbf{k}_{\perp}\right)^{2}+m^{2}}{(1-x) P^{+}},(1-x) \mathbf{P}_{\perp}-\mathbf{k}_{\perp}\right) \\
p_{1}^{\prime}=\left(x P^{\prime+}, \frac{\mathbf{k}_{\perp}^{\prime \prime}+m^{2}}{x P^{+}}, \mathbf{k}_{\perp}^{\prime}\right) \\
P_{\gamma}=\left(P^{+}, \frac{q^{2}+\mathbf{q}_{\perp}^{2}}{P^{+}}, \mathbf{P}_{\perp}\right) \\
P_{\pi}^{\prime}=\left(P^{\prime+}, \frac{M^{2}}{P^{\prime+}}, \mathbf{0}_{\perp}\right) \\
q=\left(0, \frac{Q^{2}}{P^{+}}, \mathbf{q}_{\perp}\right)
\end{array}\right.
$$


in which $\mathbf{P}_{\perp}=-\mathbf{q}_{\perp}$, and $\mathbf{k}_{\perp}^{\prime}=\mathbf{k}_{\perp}+(1-x) \mathbf{q}_{\perp}$.

$$
F_{\gamma \gamma^{*} \rightarrow \pi}\left(Q^{2}\right)=-4 \sqrt{3}\left(e_{u}^{2}-e_{d}^{2}\right) \int_{0}^{1} \mathrm{~d} x \int \frac{\mathrm{d}^{2} \mathbf{k}_{\perp}}{16 \pi^{3}} \varphi_{\pi}\left(x, \mathbf{k}_{\perp}^{\prime}\right) \frac{m}{x \sqrt{m^{2}+\mathbf{k}_{\perp}^{\prime 2}}} \frac{1}{D},
$$

where

$$
\begin{aligned}
D & =P_{\perp}^{2}-\frac{\left(x \mathbf{P}_{\perp}+\mathbf{k}_{\perp}\right)^{2}+m^{2}}{x}-\frac{\left((1-x) \mathbf{P}_{\perp}-\mathbf{k}_{\perp}\right)^{2}+m^{2}}{(1-x)} \\
& =-\frac{\mathbf{k}_{\perp}^{2}+m^{2}}{x}-\frac{\mathbf{k}_{\perp}^{2}+m^{2}}{(1-x)} .
\end{aligned}
$$

Therefore, it is obvious that $F_{\gamma \gamma^{*} \rightarrow \pi}\left(Q^{2}\right)$ we have got is frame invariant. In the first frame, we employed the Drell-Yan-West formula in the process of obtaining $F_{\gamma \gamma^{*} \rightarrow \pi}\left(Q^{2}\right)$, while in the other frame we did not use the Drell-Yan-West formula. We want to emphasize that the Drell-Yan-West formula is more convenient and simple although these two methods can give the same results.

Furthermore, we can obtain the $\gamma^{*} \gamma^{*} \rightarrow \pi^{0}$ transition form factor by substituting a virtual photon $\gamma^{*}$ for the on-shell photon $\gamma$, which means to substitute $-Q^{\prime 2}$ for $\lambda^{2}$ and gives:

$$
\begin{aligned}
F_{\gamma^{*} \gamma^{*} \rightarrow \pi^{0}}\left(Q^{2}, Q^{\prime 2}\right)= & 4 \sqrt{3}\left(e_{u}^{2}-e_{d}^{2}\right) \int_{0}^{1} \mathrm{~d} x \int \frac{\mathrm{d}^{2} \mathbf{k}_{\perp}}{16 \pi^{3}} \varphi_{\pi}\left(x, \mathbf{k}_{\perp}^{\prime}\right) \frac{m}{x \sqrt{m^{2}+\mathbf{k}_{\perp}^{\prime 2}}} \\
& \times\left(\frac{1}{Q^{\prime 2}+\frac{m^{2}+\mathbf{k}_{\perp}^{2}}{x}+\frac{m^{2}+\mathbf{k}_{\perp}^{2}}{1-x}}\right) .
\end{aligned}
$$

\section{NUMERICAL CALCULATIONS}

In the formula of the transition form factor $F_{\gamma \gamma^{*} \rightarrow \pi}\left(Q^{2}\right)$, the parameters are the normalization constant $A$, the harmonic scale $\beta$, and the quark masses $m$. In order to take a numerical calculation of the transition form factor $F_{\gamma \gamma^{*} \rightarrow \pi}\left(Q^{2}\right)$ and compare it with the available experimental data, we need to employ three constraints to fix those three parameters above. Thus, we can determine all these three parameters in the transition form factor uniquely.

1. The weak decay constant $f_{\pi}=92.4 \mathrm{MeV}$ defined 27\| from $\pi \rightarrow \mu \nu$ decay, thus one can

obtain Eq. (44) and Eq. (45) from our former work [9, 11] or Hwang's paper 8] respectively:

$$
\frac{f_{\pi}}{2 \sqrt{3}}=\int_{0}^{1} d x \int \frac{d^{2} \mathbf{k}_{\perp}}{16 \pi^{3}} \frac{\left(k_{1}^{+}+m\right)\left(k_{2}^{+}+m\right)-\mathbf{k}_{\perp}^{2}}{\left[\left(k_{1}^{+}+m\right)^{2}+\mathbf{k}_{\perp}^{2}\right]^{1 / 2}\left[\left(k_{2}^{+}+m\right)^{2}+\mathbf{k}_{\perp}{ }^{2}\right]^{1 / 2}} \varphi_{\pi}\left(x, \mathbf{k}_{\perp}\right),
$$




$$
\frac{f_{\pi}}{2 \sqrt{3}}=\int_{0}^{1} d x \int \frac{d^{2} \mathbf{k}_{\perp}}{16 \pi^{3}} \frac{m}{\sqrt{m^{2}+\mathbf{k}_{\perp}^{2}}} \varphi_{\pi}\left(x, \mathbf{k}_{\perp}\right) .
$$

One can prove that Eq. (44) is identical to Eq. (45) after some deduction, by using $q_{i}^{+}=x_{i} M$ and $M=\left(m^{2}+\mathbf{k}_{\perp}^{2}\right) / x(1-x)[9]$.

2. The charged mean square radius of $\pi^{+}$is defined as:

$$
\left\langle r_{\pi^{+}}^{2}\right\rangle=-\left.6 \frac{\partial F_{\pi^{+}}\left(Q^{2}\right)}{\partial Q^{2}}\right|_{Q^{2}=0}
$$

We can find the experimental value of $\left\langle r_{\pi^{+}}^{2}\right\rangle=0.439 \pm 0.003 \mathrm{fm}^{2} .[28]$.

3. The decay width $\Gamma\left(\pi^{0} \rightarrow \gamma \gamma\right)$ has the following relationship with $F_{\pi \gamma \gamma}(0)$ and $F_{\gamma \gamma^{*} \pi}(0)$ 24]:

$$
\left|F_{\gamma \gamma^{*} \rightarrow \pi}(0)\right|^{2}=\left|F_{\pi \rightarrow \gamma \gamma}(0)\right|^{2}=\frac{64 \pi \Gamma\left(\pi^{0} \rightarrow \gamma \gamma\right)}{(4 \pi \alpha)^{2} M_{\pi}^{3}},
$$

and we could use $\Gamma\left(\pi^{0} \rightarrow \gamma \gamma\right)=7.74 \pm 0.54 \mathrm{eV}$ [24], which leads to $F_{\pi \gamma \gamma}(0)=0.27 \pm$ $0.01 \mathrm{GeV}^{-1}$ in our calculation.

Therefore, we can obtain $m=200 \mathrm{MeV}$ (e.g., for the up quark or the down quark, assuming $\left.m_{u}=m_{d}=m\right), \beta=410 \mathrm{MeV}$, and $A=0.0475 \mathrm{MeV}^{-1}$. Reversely, we can compute the values of $f_{\pi},\left\langle r_{\pi^{+}}^{2}\right\rangle$, and $\Gamma(\pi \rightarrow \gamma \gamma)$ by using the above four parameters:

$$
\begin{aligned}
f_{\pi} & =92.4 \mathrm{MeV}, \\
\left\langle r_{\pi^{+}}^{2}\right\rangle & =0.441 \mathrm{fm}^{2}, \\
F_{\pi \rightarrow \gamma \gamma}(0) & =0.271 \mathrm{GeV}^{-1}, \\
\Gamma(\pi \rightarrow \gamma \gamma) & =7.56 \mathrm{eV} .
\end{aligned}
$$

The results are in good agreement with the experimental data which we have listed above. Moreover, it is interesting to notice that the masses of the light-flavor quarks (the up and down quarks) from the above constrains are just in the correct range (e.g., $200 \sim 300 \mathrm{MeV})$ of the constituent quark masses from more general considerations. Naturally, the transition form factor results emerging from this assumption are in quite good agreement with the experimental data.

Fig. 1 indicates that the theoretical values of the photon-pion $\left(\gamma \gamma^{*} \rightarrow \pi\right)$ transition form factors fit the experimental data well, especially in the case of low $Q^{2}$. One may consider this work as a light-cone version of relativistic quark model [9, 11, 30], which should be valid 


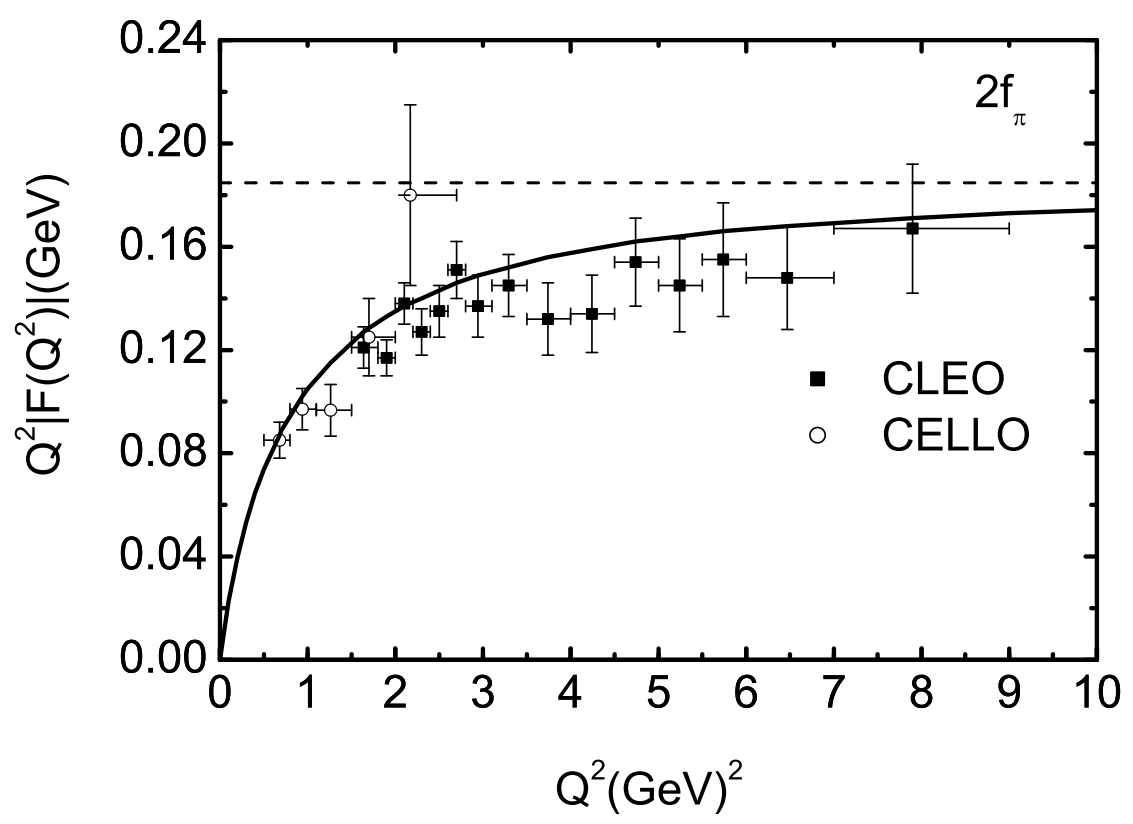

FIG. 1: Theoretical prediction for the results of $Q^{2}\left|F_{\gamma \gamma^{*} \rightarrow \pi}\left(Q^{2}\right)\right|$ calculated with the pion wave function in the BHL prescription compared with the experimental data. The data for the transition form factor are taken from Ref. [24] and Ref. [25].

in the low-energy scale about $Q^{2} \leq 2 \mathrm{GeV}^{2}$. However, it is also physically in accordance with the light-cone perturbative QCD approach [4, 5], which is applicable at the high-energy scale of $Q^{2}>2 \mathrm{GeV}^{2}$. The reason is that the hard-gluon exchange between the quark and the antiquark of the meson, which should be generally considered at high $Q^{2}$ for exclusive processes, is not necessary to be incorporated in the light-cone perturbative QCD approach for the pion-photon transition form factor [4, 5]. As a result, there is no wonder that our predictions for the transition form factor at high $Q^{2}$ also agree with the experimental data at high energy scale. Moreover, our numerical result fits the perturbative QCD result well in the limit $Q^{2} \rightarrow \infty$ which was first given by Brodsky and Lepage [4, 29]. Brodsky and Lepage found that

$$
\lim _{Q^{2} \rightarrow \infty} Q^{2}\left|F_{\gamma \gamma^{*} \rightarrow \pi}\left(Q^{2}\right)\right|=2 f_{\pi}
$$

in which $f_{\pi}$ is the decay constant. This result predicts that any mesonic wave function evolves to asymptotic wave function in the limit $Q^{2} \rightarrow \infty$. To describe the soft nonperturbative region of $Q^{2}$ with a simple interpolation between $Q^{2} \rightarrow \infty$ and $Q^{2} \rightarrow 0$ limits, they have 
proposed

$$
F_{\gamma \gamma^{*} \rightarrow \pi}\left(Q^{2}\right)=\frac{1}{4 \pi^{2} f_{\pi}} \frac{1}{1+\frac{Q^{2}}{8 \pi^{2} f_{\pi}^{2}}}
$$

in [29]. We also discover that our numerical prediction for $Q^{2}\left|F_{\gamma \gamma^{*} \rightarrow \pi}\left(Q^{2}\right)\right|$ is quite consistent with the numerical result [24] of Eq. (53), see e.g., Fig. 21 of [24].

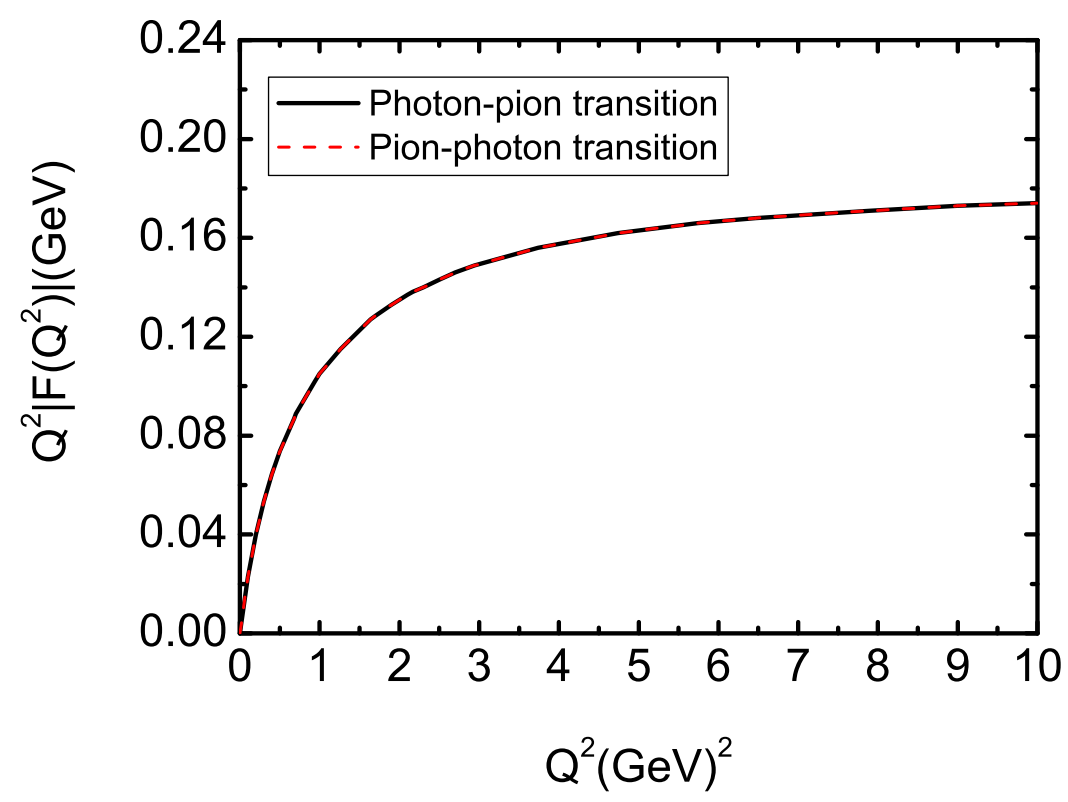

FIG. 2: Numerical results of $\gamma \gamma^{*} \rightarrow \pi$ transition form factor compared with the $\gamma^{*} \pi \rightarrow \gamma$ transition form factor, represented by the dashed and solid lines respectively.

To illustrate the intrinsic relation between the $\gamma \gamma^{*} \rightarrow \pi$ and the $\gamma^{*} \pi \rightarrow \gamma$ transition form factors, we also carry out the numerical calculation of $F_{\gamma^{*} \pi \rightarrow \gamma}\left(Q^{2}\right)$ compared with the numerical results of $F_{\gamma \gamma^{*} \rightarrow \pi}\left(Q^{2}\right)$, as shown in Fig. 2. We find an interesting result that $F_{\gamma^{*} \pi \rightarrow \gamma}\left(Q^{2}\right)$ is equal to $F_{\gamma \gamma^{*} \rightarrow \pi}\left(Q^{2}\right)$ when $Q^{2}$ varies from $0 \mathrm{GeV}^{2}$ to $10 \mathrm{GeV}^{2}$. Although $F_{\gamma \gamma^{*} \rightarrow \pi}\left(Q^{2}\right)$ and $F_{\gamma^{*} \pi \rightarrow \gamma}\left(Q^{2}\right)$ are physically different in the formulae as we have shown above, Fig. 2 indicates that they are numerically identical at very high precision, which imply that $F_{\gamma \gamma^{*} \rightarrow \pi}\left(Q^{2}\right)$ and $F_{\gamma^{*} \pi \rightarrow \gamma}\left(Q^{2}\right)$ have the same $Q^{2}$ dependence. The identification of Eq. (32) and Eq. (39) can be proved by making the variable transformation: $\mathbf{k}_{\perp} \rightarrow \mathbf{k}_{\perp}-(1-x) \mathbf{q}_{\perp}$ and then $\mathbf{q}_{\perp} \rightarrow-\mathbf{q}_{\perp}$ for Eq. (39). In fact, the identification of the two form factors can be understood from time-reverse invariance as well as parity symmetry for QED and QCD processes. Therefore, we can safely arrive at another important conclusion that $F_{\gamma^{*} \gamma^{*} \rightarrow \pi}\left(Q^{2}, Q^{\prime 2}\right)$ and $F_{\gamma^{*} \pi \rightarrow \gamma^{*}}\left(Q^{2}, Q^{\prime 2}\right)$ should be numerically identical as well as having the same $Q^{\prime 2}$ dependence, 
unless novel new physics beyond QED and QCD will be involved. This conclusion is consis-

tent with the results in [5] (for $F_{\gamma^{*} \pi \rightarrow \gamma^{*}}\left(Q^{2}, Q^{\prime 2}\right)$ ) and [8] (for $F_{\gamma^{*} \gamma^{*} \rightarrow \pi}\left(Q^{2}, Q^{\prime 2}\right)$ ) when taking the $Q^{2} \rightarrow \infty$ and $Q^{\prime 2} \rightarrow \infty$ limits,

$$
F_{\gamma^{*} \pi \rightarrow \gamma^{*}}\left(Q^{2}, Q^{\prime 2}\right)=F_{\gamma^{*} \gamma^{*} \rightarrow \pi}\left(Q^{2}, Q^{\prime 2}\right)=4 \sqrt{3}\left(e_{u}^{2}-e_{d}^{2}\right) \int_{0}^{1} \mathrm{~d} x \phi_{\pi}(x) \frac{1}{x Q^{\prime 2}+(1-x) Q^{2}} .
$$

\section{CONCLUSIONS}

The light-cone formalism provides a convenient framework for the relativistic description of hadrons in terms of quark and gluon degrees of freedom, and for the application of perturbative QCD to exclusive processes. In the application of the light-cone quark model, we obtain the minimal Fock-state expansions of the pion and the photon wave functions from the light-cone representation of the spin structure of pion and photon vertexes, then we investigate the pion-photon and the photon-pion transition form factors of the processes $\gamma^{*} \pi^{0} \rightarrow \gamma$ and $\gamma^{*} \gamma \rightarrow \pi^{0}$ by employing the quark-antiquark wave functions of the pion and the photon that we have obtained. We employ the experimental values of the pion decay constant $f_{\pi}$, the electromagnetic charged mean squared radius $\left\langle r_{\pi^{+}}^{2}\right\rangle$, and the decay width $\Gamma\left(\pi^{0} \rightarrow \gamma \gamma\right)$ as the constraints to fix those three parameters in the pion wave function. With the fixed pion wave function, we find that our numerical prediction for the $\gamma^{*} \gamma \rightarrow \pi^{0}$ transition form factor agrees with the experimental data at low and moderately high energy scale. Furthermore, we make the numerical comparison between the transition form factors of $\gamma^{*} \pi^{0} \rightarrow \gamma$ and $\gamma^{*} \gamma \rightarrow \pi^{0}$, which gives the result that these two transition form factors are equal and indicates that these two processes have intrinsic relation while they are physically different. In addition, we also give the formulae of $F_{\gamma^{*} \gamma^{*} \rightarrow \pi}\left(Q^{2}, Q^{\prime 2}\right)$ and $F_{\gamma^{*} \pi \rightarrow \gamma^{*}}\left(Q^{2}, Q^{\prime 2}\right)$, and find that they should have the same $Q^{2}$ and $Q^{\prime 2}$ dependence, unless novel new physics beyond QED and QCD will be involved.

ACKNOWLEDGMENTS This work is partially supported by National Natural Science Foundation of China under Grant Numbers 10025523 and 90103007. It is also supported by Hui-Chun Chin and Tsung-Dao Lee Chinese Undergraduate Research Endowment (CURE) at Peking University.

\section{APPENDIX: DRELL-YAN-WEST ASSIGNMENT FOR SPIN PART}

In this appendix, we prove the applicability of the Drell-Yan-West assignment to the spin part as well as for the denominator. Supposing $P$ is the momentum of the incident particle 
at rest, $P^{\prime}$ is the momentum of the final particle, $p_{1}$ and $p_{2}$ are for quarks, then $p_{1}$ is struck by $q$ into $p_{1}^{\prime}$, so we have

$$
\left\{\begin{array}{l}
p_{1}^{\prime}=p_{1}+q \\
p_{2}^{\prime}=p_{2} \\
P^{\prime}=P+q
\end{array}\right.
$$

The Drell-Yan-West assignment is to change the kinematics for the final particle from a moving frame to its rest frame:

$$
\left\{\begin{aligned}
p_{1}^{\prime} & \rightarrow p_{1}+q-x q=p_{1}+(1-x) q \\
p_{2}^{\prime} & \rightarrow p_{2}-(1-x) q \\
P^{\prime} & \rightarrow P
\end{aligned}\right.
$$

For the denominators, the general time-ordered field theory framework and the Drell-YanWest assignment give the same results respectively:

$$
\begin{aligned}
& D=\lambda^{2}+\mathbf{q}_{\perp}^{2}-\frac{m^{2}+\left(\mathbf{k}_{\perp}+\mathbf{q}_{\perp}\right)^{2}}{x}-\frac{m^{2}+\mathbf{k}_{\perp}^{2}}{1-x}, \\
& D=\lambda^{2}-\frac{m^{2}+\mathbf{k}_{\perp}^{\prime 2}}{x}-\frac{m^{2}+\mathbf{k}_{\perp}^{\prime 2}}{1-x}
\end{aligned}
$$

where $\mathbf{k}_{\perp}^{\prime}=\mathbf{k}_{\perp}+(1-x) \mathbf{q}_{\perp}$.

For the spin part, we can prove that the general field theory framework and the Drell-YanWest assignment present the identical formulae, and the assignment is more straightforward to obtain the results. Taking the calculation of $\left|\Psi_{\pi}\left(P^{+}, \mathbf{P}_{\perp}\right)\right\rangle$ for example in the general framework:

$$
\frac{\bar{u}\left(p_{1}^{+}, p_{1}^{-}, \mathbf{k}_{\perp}+\mathbf{q}_{\perp}\right)}{\sqrt{p_{1}^{+}}} \gamma_{5} \frac{v\left(p_{2}^{+}, p_{2}^{-},-\mathbf{k}_{\perp}\right)}{\sqrt{p_{2}^{+}}}
$$

we obtain

$$
\left\{\begin{array}{l}
\frac{\bar{u}_{\uparrow}}{\sqrt{p_{1}^{+}}} \gamma_{5} \frac{v_{\downarrow}}{\sqrt{p_{2}^{+}}}=+\frac{2 m P^{+}}{4 m x(1-x) P^{+2}}, \\
\frac{\bar{u}_{\downarrow}}{\sqrt{p_{1}^{+}}} \gamma_{5} \frac{v_{\uparrow}}{\sqrt{p_{2}^{+}}}=-\frac{2 m P^{+}}{4 m x(1-x) P^{+2}}, \\
\frac{\bar{u}_{\uparrow}}{\sqrt{p_{1}^{+}}} \gamma_{5} \frac{v_{\uparrow}}{\sqrt{p_{2}^{\prime+}}}=-\frac{2\left(k_{L}+(1-x) q_{L}\right) P^{+}}{4 m x(1-x) P^{+2}}, \\
\frac{\bar{u}_{\downarrow}}{\sqrt{p_{1}^{+}}} \gamma_{5} \frac{v_{\downarrow}}{\sqrt{p_{2}^{+}}}=-\frac{2\left(k_{R}+(1-x) q_{R}\right) P^{+}}{4 m x(1-x) P^{+2}},
\end{array}\right.
$$

which is equivalent to the Drell-Yan-West assignment of making the substitution of $\mathbf{k}_{\perp}^{\prime}=$ 
$\mathbf{k}_{\perp}+(1-x) \mathbf{q}_{\perp}$ for $\mathbf{k}_{\perp}$

[1] S.J. Brodsky, in Lectures on Lepton Nucleon Scattering and Quantum Chromodynamics, edited by A. Jaffe, D. Ruelle (Birkhāuser, Boston, 1982), p.255; S.J. Brodsky and G.P. Lepage, in Perturbative Quantum Chromodynamics, edited by A.H. Mueller, (World Scientific, Singapore, 1989), p.93; S.J. Brodsky, H-C. Pauli, and S.S. Pinsky, Phys. Rep. 301, 299 (1998).

[2] S.J. Brodsky and S.D. Drell, Phys. Rev. D 22, 2236 (1980).

[3] S.J. Brodsky, D.S. Hwang, B.-Q. Ma, and I. Schmidt, Nucl. Phys. B 593, 311 (2001).

[4] G.P. Lepage and S.J. Brodsky, Phys. Rev. D 22, 2157 (1980).

[5] F.-G. Cao, T. Huang, and B.-Q. Ma, Phys. Rev. D 53, 6582 (1996).

[6] P. Kroll and M. Raulfs, Phys. Lett. B 387, 848(1996).

[7] A.V. Radyushkin and R. Ruskov, Phys. Lett. B 374, 173 (1996).

[8] C.-W. Hwang, Eur. Phys. J. C 19, 105 (2001); H.M. Choi and C.R. Ji, Nucl. Phys. A 618, 291 (1997).

[9] B.-Q. Ma, Z. Phys. A 345, 321 (1993).

[10] T. Huang, B.-Q. Ma, and Q.-X. Shen, Phys. Rev. D 49, 1490 (1994).

[11] B.-W. Xiao, X. Qian, and B.-Q. Ma, Eur. Phys. J. A 15, 523 (2002), in which $q_{i}^{R, L}$ should be $q_{i}^{R, L}=q_{i}^{1} \pm \mathrm{i} q_{i}^{2}$.

[12] M.V. Terent'ev, Yad. Fiz. 24, 207 (1976) [Sov. J. Nucl. Phys. 24, 106 (1976)].

[13] V.A. Karmanov, Nucl. Phys. B 166, 378 (1980).

[14] P.L. Chung, F. Coester, and W.N. Polyzou, Phys. Lett. B 205, 545 (1988).

[15] E. Wigner, Ann. Math. 40, 149 (1939).

[16] H.J. Melosh, Phys. Rev. D 9, 1095 (1974).

[17] B.-Q. Ma, J.Phys.G: Nucl. Part. Phys.17, L53 (1991); B.-Q. Ma and Q.-R. Zhang, Z. Phys. C 58, 479 (1993).

[18] B.-Q. Ma and T. Huang, J. Phys. G: Nucl. Part. Phys. 21, 765 (1995).

[19] F.-G. Cao, J. Cao, T. Huang, and B.-Q. Ma, Phys. Rev. D 55, 7107 (1997).

[20] B.-Q. Ma, Phys. Lett. B 375, 320 (1996); B.-Q. Ma and A. Schäfer, Phys. Lett. B 378, 307 (1996); B.-Q. Ma, I. Schmidt, and J. Soffer, Phys. Lett. B 441, 461 (1998); B.-Q. Ma, I. Schmidt, and J.-J. Yang, Eur. Phys. J. A 12, 353 (2001). 
[21] B.-Q. Ma and I. Schmidt, Phys. Rev. D 58, 096008 (1998).

[22] S.J. Brodsky, T. Huang, and G.P. Lepage, in Particles and Fields-2, Proceedings of the Banff Summer Institute, Banff, Alberta, 1981, edited by A.Z. Capri and A.N. Kamal (Plenum, New York,1983), p. 143.

[23] S.D. Drell and T.-M. Yan, Phys. Rev. Lett. 24, 181 (1970); G. West, Phys. Rev. Lett. 24, 1206 (1970).

[24] CLEO Collaboration, J. Gronberg rt al., Phys. Rev. D 57, 33 (1998).

[25] CELLO Collaboration, H.-J. Behrend et al., Z. Phys. C 49, 401(1991).

[26] M. Sawicki, Phys. Rev. D 46, 474 (1992).

[27] C. Caso et al., Eur. Phys. J. C 3, 1 (1998).

[28] E.B. Dally et al., Phys. Rev. Lett. 48, 375 (1982).

[29] S.J. Brodsky and G.P. Lepage, Phys. Rev. D 24, 1808 (1981).

[30] See, e.g., L.A. Kondratyuk and M.V. Terent'ev, Yad.Fiz 31, 1087 (1980) [ Sov. J. Nucl. Phys. 31, 561 (1980)]; P.L. Chung, F. Coester, B.D. Keister, and W.N. Polyzou, Phys. Rev. C 37, 2000 (1988); H.J. Weber, Ann. Phys. (N.Y.) 207, 417 (1991); W. Jaus, Phys. Rev. D 41, 3394 (1990); D 44, 2851 (1991); P.L. Chung and F. Coester, Phys. Rev. D 44, 229 (1991); F. Schlumpf, Phys. Rev. D 48, 4478 (1993); F. Schlumpf and S.J. Brodsky, Phys. Lett. B 360, 1 (1995); B.-Q. Ma, D. Qing, and I. Schmidt, Phys. Rev. C 65, 035205 (2002). 